\title{
SÍNTESE E AVALIAÇÃO DA ATIVIDADE FITOTÓXICA DE DERIVADOS DA $\alpha$-SANTONINA
}

Elson S. Alvarenga*, Luiz C. A. Barbosa, William A. Saliba, Francisco F. P. Arantes e Antônio J. Demuner

Departamento de Química, Universidade Federal de Viçosa, 36570-000 Viçosa - MG, Brasil

Antônio A. Silva

Departamento de Fitotecnia, Universidade Federal de Viçosa, 36570-000 Viçosa - MG, Brasil

Recebido em 13/5/08; aceito em 12/9/08; publicado na web em 2/2/09

SYNTHESIS AND EVALUATION OF THE PHYTOTOXIC ACTIVITY OF $\alpha$-SANTONIN DERIVATIVES. Mixtures of $\alpha$-Santonin and various solvents were irradiated by either high or low pressure mercury lamps. The photochemical reactions afforded lumisantonin (11) $(76 \%$ in acetonitrile), (3S,3aS,9bS)-3,6,6-trimethyl-3,3a,4,5-tetrahydronafto[1,2-b]furan-2,7(6H,9bH)dione (12) $(100 \%$ in acetonitrile), $10 \alpha$-acetoxy-3-oxo-1,7 $\alpha \mathrm{H}, 6,11 \beta \mathrm{H}$-guaia-4-en-6,12-olide (8) (26\% in acetic acid), 10 $\alpha$-hydroxy-3-oxo-1,7 $\alpha \mathrm{H}, 6,11 \beta \mathrm{H}$ guaia-4-en-6,12-olide (10) (32\%) and $(E)-3-((3 S, 3 \mathrm{a} S, 7 \mathrm{a} S)-3-$ methyl-2-oxo-6-(propan-2-ylidene)hexahydrobenzofuran-7-(7aH)ylidene)propanoic acid (9) (44\%) (in water/ acetic acid 1:1, v/v). Lactone 12 was also prepared by irradiation of lumisantonin in diethyl ether. Lactones 8 and 10 were converted, respectively, into the $10 \alpha$-acetoxy-3 $\beta$-hydroxy-1,7 $\alpha \mathrm{H}, 6,11 \beta \mathrm{H}$-guaia-4-en-6,12-olide (13) $(87 \%)$ and $3 \beta, 10 \alpha$-dihydroxy-1,7 $\alpha \mathrm{H}, 6,11 \beta \mathrm{H}$-guaia-4-en-6,12-olide (14) (75\%) by sodium borohydride reduction. The effects of the compounds on the development of radicle of Sorghum bicolor and Cucumis sativus were evaluated.

Keywords: $\alpha$-santonin; photochemistry; herbicide.

\section{INTRODUÇÃO}

Nos últimos 60 anos, a indústria de agroquímicos vem constantemente disponibilizando no mercado novos compostos cada vez mais seletivos, eficientes e seguros sob o ponto de vista ambiental. ${ }^{1}$

Produtos naturais têm sido muito utilizados no desenvolvimento de herbicidas com novos mecanismos de ação, uma vez que fitotoxinas naturais apresentam freqüentemente sítios de ação diferentes dos que são alvos comuns de herbicidas sintéticos. As fitotoxinas naturais apresentam ampla diversidade estrutural incluindo, entre outros, sesquiterpenos, compostos fenólicos, substâncias heterocíclicas. ${ }^{1-4}$

As lactonas sesquiterpênicas representam uma classe de produtos naturais encontrados em plantas da família Asteraceae. Mais de 7000 estruturas já foram descritas, incluindo, germacranolídeos, eudesmanolídeos, pseudoguainolídeos e guaianolídeos. ${ }^{5}$ Essas lactonas apresentam amplo espectro de atividades biológicas, incluindo atividades fitotóxica, inseticida e fungicida. ${ }^{1}$ Como exemplos de lactonas sesquiterpênicas que apresentam atividade fitotóxica podemse citar os guaianolídeos $\mathbf{1}, \mathbf{2}$ e $\mathbf{3}$ provenientes de folhas de girassol (Figura 1). Os compostos 1 e $\mathbf{2}$ mostraram alta atividade inibitória sobre a germinação das sementes de alface (Lactuca sativa) e pouco ou nenhum efeito sobre o desenvolvimento das raízes e das partes aéreas dessa planta. Por outro lado, o composto $\mathbf{3}$, que possui três carbonilas $\alpha, \beta$-insaturadas, estimulou a germinação da alface e inibiu o crescimento das raízes e da parte aérea da alface. ${ }^{6}$

Outros exemplos de lactonas com atividade fitotóxica são os germacranolídeos 4, 6 e 7 (Figura 2). O composto 4, derivado do produto natural denominado Glaucolídeo B (5), causou forte inibição da germinação da espécie dicotiledônea Physalis ixocarpa $(90 \%$ de inibição na concentração de $2 \times 10^{-4} \mathrm{M}$ ). Já os compostos 6 e 7, nessa mesma concentração, causaram inibição moderada da germinação da monocotiledônea Lolium multiflorum, (40 e $48 \%$ de inibição, respectivamente). ${ }^{7}$

Dando continuidade aos nossos esforços para o desenvolvimento

*e-mail: elson@ufv.br

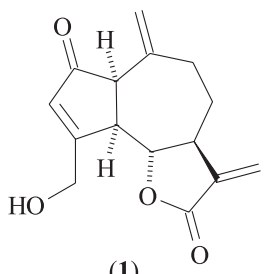

(1)

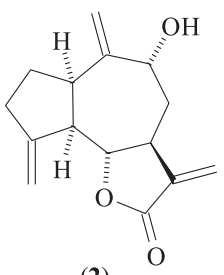

(2)<smiles>C=C1C(=O)O[C@@H]2[C@@H]3C(C)=C(O)C(=O)[C@@H]3[C@H](C)CC(OC(=O)/C(C)=C\C)[C@@H]12</smiles>

Figura 1. Exemplos de guaianolídeos que apresentam atividade fitotóxica

de novas substâncias com potencial uso como agroquímicos ${ }^{8}$ descrevemos neste trabalho a preparação de lactonas sesquiterpênicas derivadas da $\alpha$-santonina, um sesquiterpeno isolado de Artemisia santonica ${ }^{9}$ Reportamos também a avaliação da atividade fitotóxica destas lactonas sobre plantas de pepino (Cucumis sativus) e sorgo (Sorghum bicolor).

\section{PARTE EXPERIMENTAL}

As temperaturas de fusão foram determinadas em aparelho MQAPF-301 e não foram corrigidas. Os espectros na região do infravermelho foram obtidos em espectrômetro Perkin Elmer FTIR 1000, na região de 4000 a $500 \mathrm{~cm}^{-1}$, sendo as amostras preparadas na forma de pastilhas de $\mathrm{KBr}(1 \% \mathrm{~m} / \mathrm{m})$. Os espectros de RMN de ${ }^{1} \mathrm{H}$ e de ${ }^{13} \mathrm{C}$ foram obtidos em espectrômetros Varian Mercury 300 (300 MHz), Bruker DPX 200 (200 MHz) ou Bruker DRX 400 (400 $\mathrm{MHz}$ ), utilizando-se $\mathrm{CDCl}_{3}$ como solvente e TMS como referência interna. Os valores das constantes de acoplamento $(J)$ são apresenta- 


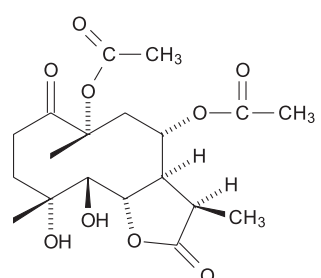

(4)

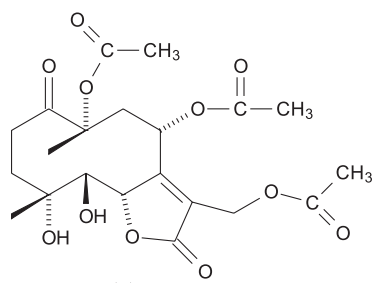

(6)

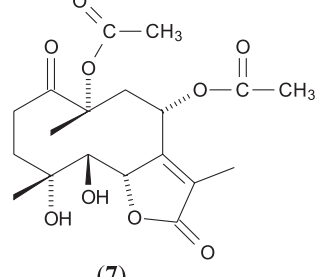

(7)

Figura 2. Exemplos de germacranolídeos que apresentam atividade fitotóxica

dos em Hz. Os espectros de massas foram obtidos em espectrômetro Shimadzu GCMS-QP5050A, por inserção direta, operando a 70 eV e faixa de varredura de 29-450 $\mathrm{Da}$. Os solventes utilizados nas reações e nas separações por cromatografia em coluna de sílica gel foram previamente purificados e secados de acordo com os procedimentos descritos na literatura. ${ }^{10}$ Todas as reações foram monitoradas por cromatografia em camada delgada utilizando-se placas de sílica gel Polygram - $\mathrm{UV}_{254} 0,25 \mathrm{~mm}$ Macherey - Nagel (MN).

\section{Procedimentos sintéticos}

10 $\alpha$-acetoxi-3-oxo-1,7 $\alpha H, 6,11 \beta H$-guaia-4-en-6,12-olídeo (8)

A um reator de borossilicato $(250 \mathrm{~mL})$ resfriado por água corrente, foram adicionados $\alpha$-santonina $(3,00 \mathrm{~g} ; 12,18 \mathrm{mmol})$ e ácido acético anidro $(150 \mathrm{~mL})$ e borbulhado nitrogênio por $30 \mathrm{~min}$. A mistura foi irradiada por uma lâmpada de vapor de mercúrio de alta pressão (125 W) durante $36 \mathrm{~h}$. O solvente foi removido sob pressão reduzida e o resíduo obtido foi dissolvido em metanol a quente $(10 \mathrm{~mL})$. Após 24 h a $-17^{\circ} \mathrm{C}$, cristais brancos da lactona $8(0,40 \mathrm{~g})$ foram obtidos por filtração a vácuo e lavados com metanol resfriado a $10^{\circ} \mathrm{C}(10 \mathrm{~mL}) . \mathrm{O}$ filtrado foi concentrado sob pressão reduzida em evaporador rotativo e o resíduo obtido foi purificado em coluna de sílica-gel (hexano/acetato de etila $2: 1, \mathrm{v} / \mathrm{v}$ ), fornecendo mais $0,47 \mathrm{~g}$ do mesmo produto (total $0,87 \mathrm{~g} ; 26 \%$ de rendimento). $\mathrm{T}_{\mathrm{f}}=174,7-175,9^{\circ} \mathrm{C} . \mathrm{IV}\left(\overline{\mathrm{v}}_{\max }, \mathrm{cm}^{-1}\right)$ : 2968, 2934, 2878, 1777, 1735, 1706, 1649, 1453, 1370, 1317, 1275, 1249, 1218, 1185, 1157, 1089, 1056, 1020, 987, 969, 951, 890, 848, 712. RMN de ${ }^{1} \mathrm{H}\left(400 \mathrm{MHz}, \mathrm{CDCl}_{3}\right): \delta$ (m, Int., $J$, atrib.): 1,09 (s, $3 \mathrm{H}, \mathrm{H} 14), 1,25$ (d, 3H, $\left.J_{13,11}=6,8, \mathrm{H} 13\right), 1,40-1,53$ (m, 1H, H8'), 1,91 (s, 3H, H15); 2,00 (s, 3H, $\mathrm{CH}_{3} \mathrm{CO}$ ); 2,05 - 2,13 (m, 1H, H8); 2,15 - 2,25 (m, 2H, H7 e H9'); 2,33 (dq, $1 \mathrm{H}, J_{11,7}=12,2, J_{11,13}=6,8$, H11); 2,40 (dd, $\left.1 \mathrm{H}, J_{2,2}=19,4, J_{2,1}=2,6, \mathrm{H} 2\right) ; 2,50$ (dd, $1 \mathrm{H}, J_{2,2}=$ $19,4, J_{2,1}=6,3, \mathrm{H} 2$ ') $; 2,62$ (dt, $1 \mathrm{H}^{2}, J_{9,}=13,5, J_{\text {vic }}=4,4, \mathrm{H} 9$ ); 4,15 $-4,17$ (m, 1H, H1); 4,81 (d, 1H, $\left.J_{67}=10,9, \mathrm{H} 6\right)$. RMN de ${ }^{13} \mathrm{C}(100$ $\left.\mathrm{MHz}, \mathrm{CDCl}_{3}\right): \delta 9,4(\mathrm{C} 15) ; 12,4(\mathrm{C} 13) ; 20,0(\mathrm{C} 14) ; 22,2\left(\mathrm{CH}_{3} \mathrm{CO}\right)$; 25,3 (C8); 36,8 (C2); 37,9 (C9); 41,3 (C11); 47,2 (C1); 48,2 (C7); 81,2 (C6); 85,5 (C10); 143,2 (C4); 160,8 (C5); 170,3 (C=O, acetato); 177,0 (C12); 206,9 (C3). EM, m/z (\%): $306\left(\mathrm{M}^{+}, \mathrm{C}_{17} \mathrm{H}_{22} \mathrm{O}_{5}, 1 \%\right), 264$ (10), 246 (20), 231 (10), 173 (7), 55 (15), 43 (100).

Ácido (E)-3-((3S,3aS,7aS)-3-metil-2-oxo-6-(propan-2-ilideno) hexa-hidrobenzo furan-7-(7aH)-ilideno)propanóico $(9) e$ 10 $\alpha$-hidroxi-3-oxo-1,7 $\alpha H, 6,11 \beta H$-guaia-4-en-6,12-olídeo (10)
A um reator de borossilicato resfriado por água corrente, contendo uma mistura de água $(90 \mathrm{~mL})$ e acetonitrila $(70 \mathrm{~mL})$ foi adicionado $\alpha$-santonina ( $1,00 \mathrm{~g} ; 4,06 \mathrm{mmol})$ e borbulhado nitrogênio por 30 min. A mistura reacional foi então irradiada com uma lâmpada de mercúrio de alta pressão $(125 \mathrm{~W})$ por $36 \mathrm{~h}$. A acetonitrila foi removida sob vácuo e a fase aquosa extraída com acetato de etila $(3 \times 70 \mathrm{~mL})$. A fase orgânica foi secada com sulfato de magnésio anidro, filtrada e concentrada sob pressão reduzida. O resíduo foi fracionado em coluna de sílica-gel (hexano/acetato de etila 1:1 v/v) fornecendo a lactona 10 (0,39 g, 37\% de rendimento) e o ácido 9 (0,13 g, 12\% de rendimento) como sólidos brancos.

Em um segundo método foi empregado como solvente da reação uma mistura de ácido acético $(70 \mathrm{~mL})$ e água $(70 \mathrm{~mL})$ sendo as outras condições idênticas ao procedimento descrito anteriormente. A lactona 10 e o ácido 9 foram obtidos em 32 e $44 \%$ de rendimento, respectivamente.

Dados referentes ao composto $(\mathbf{9}) . \mathrm{T}_{\mathrm{f}}=145,4-146,2{ }^{\circ} \mathrm{C}$. IV $\left(\overline{\mathrm{v}}_{\max }\right.$, $\left.\mathrm{cm}^{-1}\right)$ : 2983, 2932, 2879 1781, 1709, 1654, 1455, 1380, 1240, 1221, 1183, 1161, 1139, 1081, 1052, 1035, 1006, 935, 876, 743, 680, 624, 596, 513. RMN de ${ }^{1} \mathrm{H}\left(200 \mathrm{MHz}, \mathrm{CDCl}_{3}\right): \delta$ (m, Int., $J$, atrib.): 1,23 (d, 3H, $\left.J_{12,3}=6,9, \mathrm{H} 12\right) ; 1,28-1,45$ (m, 1H, H4); 1,64 (s, 3H, H13); 1,77 (d, 3H, H14); 1,77 - 1,91 (m, 2H, H3a, H5'); 1,92 - 2,06 (m, $1 \mathrm{H}, \mathrm{H} 4$ '); 2,34 (dq, $\left.1 \mathrm{H}, J_{3,3 \mathrm{a}}=12,6, J_{3,12}=6,9, \mathrm{H} 3\right) ; 2,80-2,89(\mathrm{~m}$, 1H, H5); 2,98 (ddd, $1 \mathrm{H}, J_{9,9}=17,4, J_{9,8}=7,8, J_{9,7 \mathrm{a}}=1,2, \mathrm{H} 9$ ); 3,03 (ddd, $1 \mathrm{H}, J_{9^{\prime}, 9}=17,4, J_{9^{\prime}, 8}=6,5, J_{9^{\prime}, 7 \mathrm{a}}=2,0, \mathrm{H}^{\prime}$ ) $) ; 4,13\left(\mathrm{dd}, 1 \mathrm{H}, J_{7 \mathrm{a}, 3 \mathrm{a}}\right.$ $\left.=10,6, J_{7 \mathrm{a}, 8}=1,4, \mathrm{H} 7 \mathrm{a}\right) ; 5,67\left(\mathrm{ddd}, 1 \mathrm{H}, J_{8,9}=7,8, J_{8,9}=6,5, J_{8,7 \mathrm{a}}=\right.$ 1,4, H8). RMN de ${ }^{13} \mathrm{C}\left(50 \mathrm{MHz}, \mathrm{CDCl}_{3}\right): \delta 12,5$ (C12); 20,0 (C14); 22,1 (C13); 27,4 (C4); 30,4 (C5); 33,7 (C9); 42,2 (C3); 54,2 (C3a); 83,4 (C7a); 110,4 (C8); 127,6 (C6); 131,0 (C11); 140,2 (C7); 177,5 (C2); 178,7 (C10). EM, m/z (\%): $264\left(\mathrm{M}^{+}, \mathrm{C}_{15} \mathrm{H}_{20} \mathrm{O}_{4}, 1 \%\right), 263$ (20), 218 (16), 145 (96), 73 (100), 55 (74) .

Dados referentes ao composto $(\mathbf{1 0}) . \mathrm{T}_{\mathrm{f}}=160,1-161,0{ }^{\circ} \mathrm{C}$. IV $\left(\bar{v}_{\mathrm{max}}\right.$, $\left.\mathrm{cm}^{-1}\right)$ : 3448, 2972, 2931, 2878, 1776, 1699, 1641, 1458, 1381, 1340, 1313, 1233, 1177, 1154, 1099, 1055, 1025, 991, 965, 947, 884, 735, 708, 630. RMN de ${ }^{1} \mathrm{H}\left(300 \mathrm{MHz}, \mathrm{CDCl}_{3}\right): \delta$ (m, Int., $J$, atrib.): 1,00 (s, 3H, H14); 1,31 (d, 3H, $\left.J_{13,11}=6,0, \mathrm{H} 13\right) ; 1,40-1,60$ (m, 1H, H8'); 1,70 - 1,88 (m, 1H, H9'); 1,90 (s, 3H, H15), 2,00 - 2,29; (m, 3H, $\mathrm{H} 7, \mathrm{H} 8, \mathrm{H} 9$ ); 2,32 (dq, 1H, $J_{11,7}=12,2, J_{11,13}=6,9, \mathrm{H} 11$ ); 2,50 - 2,70 (m, 2H, H2, H2'); 3,20 - 3,35 (m, 1H, H1); 4,86 (d, 1H, $J_{6,7}=11,1$, H6). RMN de ${ }^{13} \mathrm{C}$ (75 MHz, $\mathrm{CDCl}_{3}$ ): $\delta$ 9,8 (C14); 12,8 (C13); 21,5 (C15); 26,1 (C8); 37,5 (C2); 41,7 (C11); 45,5 (C9); 48,7 (C7); 50,8 (C1); 74,7 (C10); 81,8 (C6); 143,3 (C4); 161,9 (C5); 177,6 (C12); 208,4 (C3). EM, $m / z(\%): 264\left(\mathrm{M}^{+}, \mathrm{C}_{15} \mathrm{H}_{20} \mathrm{O}_{4}, 90 \%\right), 221$ (10), 206 (20), 193 (58), 123 (50), 55 (100).

\section{Lumissantonina (11)}

Nitrogênio foi borbulhado por $30 \mathrm{~min}$ em uma solução da $\alpha$-santonina $(0,5 \mathrm{~g} ; 2,03 \mathrm{mmol})$ em acetonitrila anidra $(120 \mathrm{~mL})$ em um tubo de quartzo. O tubo foi tampado e imerso em banho de água gelada mantendo a temperatura da solução constante em $15{ }^{\circ} \mathrm{C}$ durante a reação. A mistura reacional foi irradiada por 4 lâmpadas de vapor de mercúrio de baixa pressão $(4 \times 15 \mathrm{~W})$ por $6 \mathrm{~h}$. O solvente foi removido sob pressão reduzida e o resíduo recristalizado em uma mistura de hexano e acetona (aproximadamente 2:1, v/v), resultando na obtenção do composto $\mathbf{1 1}$ como um sólido amarelo palha (0,38 g, $76 \%$ de rendimento).

Em um outro experimento, mantiveram-se as mesmas condições de reação, exceto a temperatura que foi mantida a $70{ }^{\circ} \mathrm{C}$ por $2 \mathrm{~h}$ de reação. Nessas condições obteve-se a lactona (11) com rendimento de $76 \%$. $\mathrm{T}_{\mathrm{f}}=147,8-148,9^{\circ} \mathrm{C}$. IV $\left(\bar{v}_{\max }, \mathrm{cm}^{-1}\right): 2993,2943,2883,1776$, 1699, 1572, 1460, 1138, 1299, 1253, 1167, 1145, 1093, 1029, 998, 830, 742, 725, 656, 534, 495. RMN de ${ }^{1} \mathrm{H}\left(300 \mathrm{MHz}, \mathrm{CDCl}_{3}\right): \delta(\mathrm{m}$, Int., $J$, atrib.): 1,11 (s, 3H, H14); 1,12-1,21 (m, 1H, H9a); 1,22 (s, 3H, 
$\mathrm{H} 15) ; 1,25$ (d, 1H, $\left.J_{13.11}=6,9, \mathrm{H} 13\right) ; 1,57-1,69$ (m, 1H, H8); 1,79-1,98 (m, 3H, H9e, H10e, H10a); 2,30 (dq, $\left.1 \mathrm{H}, J_{11,8}=12,3, J_{11,13}=6,9, \mathrm{H} 11\right)$; $3,81\left(\mathrm{~d}, 1 \mathrm{H}, J_{7.8}=10,8, \mathrm{H} 7\right) ; 6,01,\left(\mathrm{~d}, 1 \mathrm{H}, J_{4,5}=5,7, \mathrm{H} 4\right) ; 7,59(\mathrm{~d}, 1 \mathrm{H}$, $\left.J_{5,4}=5,7, \mathrm{H} 5\right)$. RMN de ${ }^{13} \mathrm{C}\left(75 \mathrm{MHz}, \mathrm{CDCl}_{3}\right): \delta 7,7(\mathrm{C} 15) ; 12,7(\mathrm{C} 13)$; 17,5 (C14); 22,8 (C9); 29,9 (C10); 40,6 (C6); 41,5 (C11); 43,0 (C2); 49,0 (C8); 60,4 (C1); 77,9 (C7); 131,7 (C4); 158,0 (C5); 178,9 (C12); 206,9 (C3). EM, $m / z$ (\%): $246\left(\mathrm{M}^{+} . \mathrm{C}_{15} \mathrm{H}_{18} \mathrm{O}_{3}, 35 \%\right) ; 173$ (49); 145 (24); 135 (60); 107 (38); 91 (81); 77 (52); 55 (100).

(3S,3aS,9bS)-3,6,6-trimetil-3,3a,4,5-tetra-hidronafto[1,2-b]furan2,7(6H,9bH)diona (12)

A um reator de borossilicato, resfriado com um condensador de água à temperatura ambiente, adicionou-se $\alpha$-santonina $(2,0 \mathrm{~g} ; 8,61$ mmol) e acetonitrila anidra $(150 \mathrm{~mL})$. A solução foi borbulhada com nitrogênio por 30 min e irradiada por $36 \mathrm{~h}$ utilizando-se uma lâmpada de vapor de mercúrio de alta pressão $(125 \mathrm{~W})$. O solvente foi removido sob pressão reduzida e o resíduo fracionado em coluna de sílica-gel (hexano/acetato de etila 1:1, v/v) sendo obtido o composto 12 como um óleo amarelado $(0,77 \mathrm{~g} ; 39 \%$ de rendimento).

A reação foi repetida nas seguintes condições: nitrogênio foi borbulhado por 30 min em uma solução da $\alpha$-santonina (2,0 g; 8,12 $\mathrm{mmol}) \mathrm{em}$ acetonitrila anidra $(150 \mathrm{~mL}) \mathrm{em}$ um tubo de quartzo, que foi posteriormente vedado com rolha esmerilhada. A mistura reacional foi irradiada por $24 \mathrm{~h}$ por 4 lâmpadas de mercúrio de baixa pressão ( 4 x $15 \mathrm{~W})$ e o solvente removido sob vácuo. A lactona 12 foi obtida $(2,00 \mathrm{~g} ; 100 \%)$ como um óleo amarelado. IV $\left(\bar{v}_{\max }, \mathrm{cm}^{-1}\right): 2972,2935$, 2876, 1783, 1698, 1661, 1626, 1561, 1459, 1380, 1334, 1300, 1251, $1165,1139,1117,1099,1028,997,895,837,800,653,528$. RMN de ${ }^{1} \mathrm{H}\left(300 \mathrm{MHz}, \mathrm{CDCl}_{3}\right.$ ): $\delta$ (m, Int., J, atrib.): 1,17 (s, 3H, H11); 1,20 (s, $3 \mathrm{H}, \mathrm{H} 12) ; 1,30$ (d, 3H, $\left.J_{10,3}=6,9, \mathrm{H} 10\right) ; 1,50-2,00$ (m, 2H, H4, H5); 2,10 - 2,20 (m, 1H, H4'); 2,30 - 2,60 (m, 3H, H3, H3a, H5'); 3,80 (d, $\left.1 \mathrm{H}, J_{9 \mathrm{~b}, 3 \mathrm{a}}=10,6, \mathrm{H} 9 \mathrm{~b}\right) ; 6,00\left(\mathrm{~d}, 1 \mathrm{H}, J_{8,9}=9,6, \mathrm{H} 8\right) ; 7,15\left(\mathrm{~d}, 1 \mathrm{H}, J_{9,8}=\right.$ 9,6, H9). RMN de ${ }^{13} \mathrm{C}\left(75 \mathrm{MHz}, \mathrm{CDCl}_{3}\right)$ : $\delta 12,7$ (C10); 22,6 (C4); 24,7 (C11); 25,6 (C12); 26,2 (C5); 41,9 (C3); 48,7 (C3a); 50,2 (C6); 79,6 (C9b); 123,5 (C8); 125,3 (C9a); 138,7 (C9); 152,3 (C5a); 179,3 (C2); 206,0 (C7). EM, m/z (\%): $246\left(\mathrm{M}^{+}, \mathrm{C}_{15} \mathrm{H}_{18} \mathrm{O}_{3}, 2 \%\right), 55,65,77$, 91, 107, 119, 135, 147, 161, 175, 183, 203, 217, 131, 246.

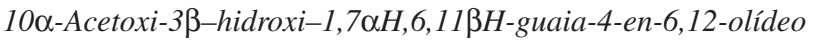
(13)

Boroidreto de sódio $(0,15 \mathrm{~g} ; 4,00 \mathrm{mmoL})$ foi adicionado à uma solução da lactona 8 (0,31 g; 1,01 mmoL) em metanol anidro (70 $\mathrm{mL}$ ). Após $2 \mathrm{~h}$ de reação sob agitação magnética, solução saturada de cloreto de amônio $(70 \mathrm{~mL})$ foi adicionada à mistura. O resíduo sólido foi removido por filtração, lavado com metanol $(10 \mathrm{~mL})$. O filtrado foi concentrado sob pressão reduzida para a remoção do metanol, sendo a fase aquosa resultante extraída com acetato de etila $(3 \times 70 \mathrm{~mL})$. A fase orgânica foi então lavada com solução saturada de cloreto de sódio $(50 \mathrm{~mL})$, secada com sulfato de magnésio anidro, filtrada e o solvente removido sob pressão reduzida. O resíduo amarelado obtido foi purificado em coluna de sílica gel (hexano/acetato de etila 1:1, v/v) fornecendo o álcool 13 como um sólido branco $(0,27 \mathrm{~g} ; 87 \%$ de rendimento). $\mathrm{T}_{\mathrm{f}}=106-107^{\circ} \mathrm{C} . \mathrm{IV}\left(\overline{\mathrm{v}}_{\max }, \mathrm{cm}^{-1}\right): 3434,2970,2933$, 2873, 1770, 1726, 1456, 1369, 1250, 1216, 1180, 1153, 1124, 1087, 1055, 1017, 987, 963, 850, 801, 755, 735, 699, 607. RMN de ${ }^{1} \mathrm{H}$ (300 MHz, $\mathrm{CDCl}_{3}$ ): $\delta$ (m, Int., $J$, atrib.): 1,16 (s, 3H, H14); 1,20 (d, $3 \mathrm{H}, J_{13,11}=7,2$, H13); 1,36 - 1,40 (m, 1H, H8'); 1,57 (ddd, 1H, $J_{2,2}$ $\left.=14,0, J_{2,1} \cong J_{2,3} \cong 6,4, \mathrm{H} 2\right) ; 1,73(\mathrm{sl}, 1 \mathrm{H}, \mathrm{OH}) ; 1,85(\mathrm{~s}, 3 \mathrm{H}, \mathrm{H} 15)$; 1,95 (s, 3H, $\mathrm{CH}_{3} \mathrm{CO}$ ); 1,99 - 2,25 (m, 4H, H7, H8, H9', H11); 2,362,48 (m, 2H, H2', H9); 3,67 - 3,80 (m, 1H, H1); 4,48 - 4,60 (m, 1H, $\mathrm{H} 3) ; 4,65$ (d, $\left.1 \mathrm{H}, J_{6,7}=10,8, \mathrm{H} 6\right)$. RMN de ${ }^{13} \mathrm{C}\left(75 \mathrm{MHz}, \mathrm{CDCl}_{3}\right)$ : $\delta$ 12,6 (C13); 12,7 (C15); 20,4 (C14); 22,7 ( $\left.\mathrm{CH}_{3} \mathrm{CO}\right) ; 25,4$ (C8); 34,8 (C2); 38,1 (C9); 41,7 (C11); 49,1 (C7); 51,4 (C1); 77,9 (C3); 81,7
(C6); 86,8 (C10); 131,3 (C5); 144,4 (C4); 170,7 (C=O, acetil); 178,5 (C12). EM, m/z (\%): $308\left(\mathrm{M}^{+}, \mathrm{C}_{17} \mathrm{H}_{24} \mathrm{O}_{5}, 1 \%\right), 248$ (17), 233 (35), 175 (15), 55 (20), 43 (100).

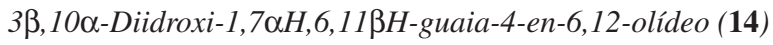

Boroidreto de sódio $(0,07 \mathrm{~g} ; 1,87 \mathrm{mmol})$ foi adicionado a uma solução da lactona $\mathbf{1 0}\left(0,120 \mathrm{~g} ; 4,54 \times 10^{-4} \mathrm{~mol}\right)$ em metanol anidro (20 $\mathrm{mL}$ ). Após $2 \mathrm{~h}$ de reação sob agitação magnética, solução saturada de cloreto de amônio $(20 \mathrm{~mL})$ foi adicionada à mistura. $\mathrm{O}$ resíduo sólido foi removido por filtração, lavado com metanol $(10 \mathrm{~mL})$ e o filtrado foi concentrado sob vácuo para a remoção do metanol. A fase aquosa foi extraída com acetato de etila $(3 \times 20 \mathrm{~mL})$, e o extrato orgânico foi lavado com solução saturada de cloreto de sódio $(20 \mathrm{~mL})$, secado com sulfato de magnésio anidro, filtrado e o solvente removido sob pressão reduzida. O resíduo oleoso obtido foi fracionado em coluna de sílica gel (hexano/acetato de etila 1:5, v/v) fornecendo o composto 14 como um sólido branco $(0,091 \mathrm{~g} ; 75 \%)$. $\mathrm{T}_{\mathrm{f}}=168,5-169,5^{\circ} \mathrm{C}$. IV $\left(\bar{v}_{\max }, \mathrm{cm}^{-1}\right): 3398,2971,2920,2851,1762,1655,1458,1379,1341$, 1319, 1262, 1234, 1210, 1179, 1132, 1103, 1077, 865, 751, 735, 669. RMN de ${ }^{1} \mathrm{H}\left(300 \mathrm{MHz}, \mathrm{CDCl}_{3}\right): \delta$ (m, Int, $J$, atrib.): 1,05 (s, $3 \mathrm{H}, \mathrm{H} 14) ; 1,20$ (d, 3H, $\left.J_{13,11}=7,0, \mathrm{H} 13\right) ; 1,30-1,45$ (m, 1H, H8'), 1,60 - 1,69 (m, 4H, 2OH, H2, H9'); 1,89 (s, 3H, H15); 1,90-2,10 (m, 3H, H7, H8, H9); 2,20 (dq, 1H, $J_{11,7}=12,2, J_{11,13}=7,0, \mathrm{H} 11$ ); $2,50\left(\mathrm{dt}, 1 \mathrm{H}, J_{2^{\prime}, 2}=16,0, J_{2^{\prime}, 3}=J_{2^{\prime}, 1}=8,0, \mathrm{H} 2{ }^{\prime}\right) ; 2,80-3,00(\mathrm{~m}, 1 \mathrm{H}$, $\mathrm{H} 1$ ); 4,40 - 4,60 (m, 1H, H3); 4,68 (d, 1H, $J_{6,7}=10,8$, H6). RMN de ${ }^{13} \mathrm{C}\left(75 \mathrm{MHz} \mathrm{CDCl}_{3}\right): \delta 12,7$ (C15); 12,7 (C13); 21,9 (C14); 25,9 (C8); 35,0 (C2); 41,7 (C11); 45,1 (C9); 49,3 (C7); 54,9 (C1); 74,8 (C10); 77,9 (C3); 82,0 (C6); 132,0 (C4); 144,2 (C5); 178,4 (C12). $\mathrm{EM}, m / z$ (\%): $266\left(\mathrm{M}^{+}, \mathrm{C}_{15} \mathrm{H}_{22} \mathrm{O}_{4}, 1 \%\right), 264$ (10), 248 (28), 193 (45), $123(60), 55$ (100).

\section{Ensaios biológicos}

Pentan-3-ona ( $24 \mu \mathrm{L})$, xileno ( $48 \mu \mathrm{L})$, monoleato de polietileno glicol (Tween $80,72 \mu \mathrm{L}$ ), água destilada $(2 \mathrm{~mL})$ e o composto avaliado $(0,02$ $\mathrm{mmol}$ ) foram adicionados a um tubo de ensaio. Após este tubo ter sido agitado vigorosamente por $1 \mathrm{~min}$, seu conteúdo foi transferido para um balão volumétrico de $50 \mathrm{~mL}$ e o volume completado com água destilada. Uma mistura de mesma composição, porém sem o composto a ser avaliado, foi utilizada como controle. O delineamento experimental foi inteiramente casualizado com 5 repetições. ${ }^{11}$ Cada repetição foi composta por uma placa de Petri ( $9 \mathrm{~cm}$ de diâmetro por $3 \mathrm{~cm}$ de altura) contendo 20 sementes de pepino (Cucumis sativus) ou de sorgo (Sorghum bicolor) mantidas sobre papel filtro embebido com $5 \mathrm{~mL}$ de solução de um dos compostos sintetizados na concentração de $4 \times 10^{-4} \mathrm{M}$.

As placas de Petri foram mantidas por $72 \mathrm{~h}$ em estufas incubadoras a $25 \pm 0,5^{\circ} \mathrm{C}$. Após esse período, contaram-se as sementes que germinaram e mediu-se o comprimento das raízes. A média do comprimento radicular e a porcentagem de inibição do crescimento das raízes foram calculadas em relação ao controle e os dados foram submetidos à análise de variância. Para comparação das médias, utilizou-se o teste de Tukey a $5 \%$ de probabilidade. ${ }^{12}$

\section{RESULTADOS E DISCUSSÃO}

\section{Síntese}

Visando a obtenção de lactonas com esqueletos guaianolídeo e eudesmanolídeo para fins de estudo de suas propriedades fitotóxicas, o produto natural $\alpha$-santonina foi submetido a reações fotoquímicas sob diferentes condições. Nesse trabalho variou-se o solvente, a temperatura, a fonte de radiação e o tipo de reator, conforme apresentado no Esquema 1. 

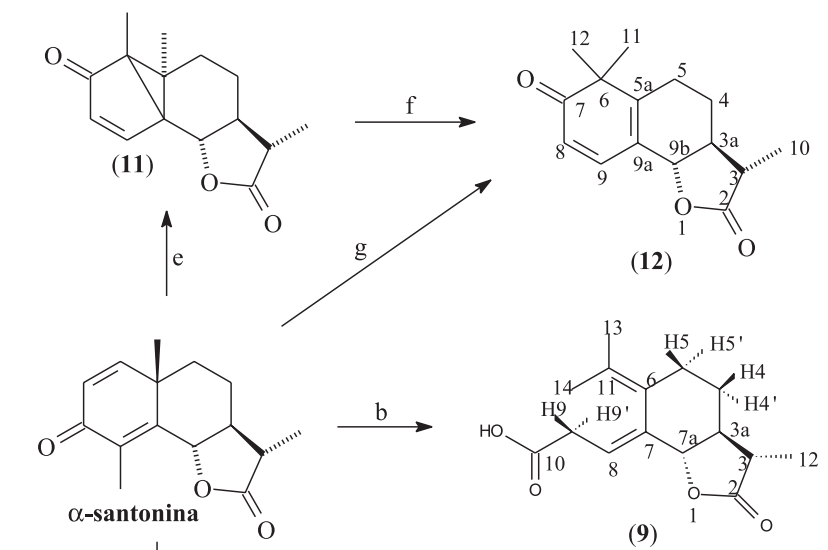<smiles>CC(=O)O[C@]1(C)CCC2[C@@H](C)C(=O)O[C@H]2C2=C(C)C(=O)CC21</smiles><smiles>CC1=C2CC[C@@](C)(O)C(=O)CC2[C@@H](C)C1=O</smiles>

$(\mathbf{1 0})$<smiles>CC1([Tl])CCCCC1</smiles>

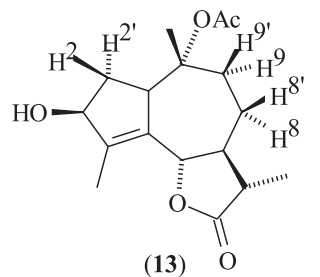

(13) $\mathrm{O}$

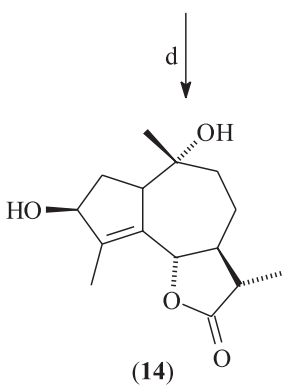

Esquema 1. a) $\mathrm{Hg}$-AP/RB ${ }^{*}, \mathrm{AcOH}, 25^{\circ} \mathrm{C}, 36 \mathrm{~h}, 26 \%$; b) $\mathrm{Hg}$ - $\mathrm{AP} / \mathrm{RB}, \mathrm{H}_{2} \mathrm{O} /$ AcOH 1:1 v/v, $25^{\circ} \mathrm{C}, 36 \mathrm{~h}$, (9) 44\%, (10) 32\%; c) $\mathrm{NaBH}_{4}, \mathrm{MeOH}, 2 \mathrm{~h}, 87 \%$; d) $\mathrm{NaBH}_{4}, \mathrm{MeOH}, 2 \mathrm{~h}, 75 \%$; e) $\mathrm{Hg}-\mathrm{BP} / \mathrm{RQ} Q^{\#}, \mathrm{MeCN}, 70^{\circ} \mathrm{C}, 2 \mathrm{~h}, 76 \%$; f) $\mathrm{Hg}$ $B P / R Q, E t_{2} O, 15^{\circ} \mathrm{C}, 12 \mathrm{~h}, 100 \%$; g) $\mathrm{Hg}-\mathrm{BP} / \mathrm{RQ}, \mathrm{MeCN}, 70^{\circ} \mathrm{C}, 24 \mathrm{~h}, 100 \%$; $H g-A P / R B$ - lâmpada de mercúrio de alta pressão/reator de borossilicato; * $H g-B P / R Q$ - lâmpada de mercúrio de baixa pressão/reator de quartzo

Como pode ser observado no Esquema 1 e na Tabela 1, a variação das condições de reação mencionadas, resultou na formação de produtos diferentes, ou em alguns casos observou-se apenas variação nos rendimentos dos compostos obtidos.

A irradiação da $\alpha$-santonina, dissolvida em ácido acético anidro em reator de borossilicato, com lâmpada ultravioleta de alta pressão, resultou na formação do composto $8 \mathrm{em} 26 \%$ de rendimento. Apesar da síntese de $\mathbf{8}$ já ter sido relatada na literatura, ${ }^{13-17}$ esse composto havia sido apenas parcialmente caracterizado e nenhum relato de suas propriedades fitotóxicas foi encontrado. Em função disso, sua obtenção foi necessária no presente estudo.

O espectro no infravermelho de $\mathbf{8}$ apresentou bandas de absorção em 1783, 1729 e $1707 \mathrm{~cm}^{-1}$, relativas aos estiramentos $\mathrm{C}=\mathrm{O}$ da lactona, do grupo acetil e da cetona, respectivamente. $\mathrm{O}$ pico do íon molecular em $m / z=306$, correspondendo à fórmula molecular $\mathrm{C}_{17} \mathrm{H}_{22} \mathrm{O}_{5}$, foi observado no espectro de massas.

Por meio da análise de experimentos bidimensionais de ressonância magnética nuclear (COSY e NOESY), foi feita a atribuição completa dos sinais de todos os átomos de hidrogênio e de carbono, o que não foi relatado em trabalhos anteriores. ${ }^{13-17}$ Essa reação foi repetida várias vezes, sempre com rendimento do produto em torno de $26 \%$.

Visando o preparo das lactonas $\mathbf{9}$ e $\mathbf{1 0}$ a partir da $\alpha$-santonina, para fins de estudo da atividade fitotóxica, empregaram-se as condições reacionais ligeiramente diferentes das relatadas na literatura. Barton et al. ${ }^{13}$ utilizaram na reação fotoquímica uma mistura de ácido acético e água $(9: 11, v / v)$, em reator de borossilicato, mantendo a temperatura de $-5 \mathrm{a}+5^{\circ} \mathrm{C}$, e irradiação por 90 min com lâmpada ultravioleta de alta pressão. Nessas condições os autores obtiveram os compostos 9 e $\mathbf{1 0}$ com rendimentos de aproximadamente $16 \%$ cada.

Neste trabalho a $\alpha$-santonina, dissolvida em uma mistura de ácido acético e água $(1: 1, \mathrm{v} / \mathrm{v})$, em um reator de borossilicato, foi irradiada com uma lâmpada de mercúrio de alta pressão por $36 \mathrm{~h}$, mantendo-se a temperatura a $25^{\circ} \mathrm{C}$. Nessas condições as lactonas $\mathbf{9}$ e $\mathbf{1 0}$ foram isoladas com rendimentos de 44 e $32 \%$, respectivamente, superiores aos relatados na literatura. ${ }^{13}$ Não foi possível isolar e caracterizar outros subprodutos dessa reação.

A caracterização dos compostos $\mathbf{9}$ e $\mathbf{1 0}$ foi feita por meio das espectroscopias no infravermelho (IV), RMN de ${ }^{1} \mathrm{H}, \mathrm{RMN}$ de ${ }^{13} \mathrm{C}$, dos experimentos em duas dimensões HMBC e NOESY e por meio da espectrometria de massas. A estereoquímica de tais compostos foi originalmente proposta com base em dados de raio-x..$^{18,19}$

Com vistas a avaliar a influência do solvente sobre o resultado da reação discutida acima, a $\alpha$-santonina foi irradiada com a lâmpada de alta pressão no reator de borossilicato, utilizando-se, os seguintes solventes: mistura de acetonitrila e água $(9: 7, \mathrm{v} / \mathrm{v})$ e acetonitrila pura (Tabela 1). Com acetonitrila e água foram obtidas as lactonas $10(37 \%)$ e 9 (12\%), enquanto que com o uso de acetonitrila anidra apenas o composto 12 foi isolado em $39 \%$ de rendimento.

A formação dos guaianolídeos $\mathbf{8}$ e $\mathbf{1 0}$ pode ser explicada, mecanisticamente, pelo ataque nucleofílico do ácido acético ou da água ao carbono C-10 da $\alpha$-santonina. ${ }^{20,21}$

Visando ainda avaliar a influência da fonte de radiação sobre os produtos da reação, a $\alpha$-santonina dissolvida em acetonitrila, em um tubo de quartzo, foi irradiada com lâmpada de mercúrio de baixa

Tabela 1. Condições e rendimentos das reações fotoquímicas da $\alpha$-santonina e da lumissantonina

\begin{tabular}{lcccccc}
\hline Lâmpada de mercúrio & Material de partida & Reator & Solvente & Temp. $\left({ }^{\circ} \mathrm{C}\right)$ & Tempo (h) & Rendimento (Produto) \\
\hline Alta Pressão & $\alpha$-santonina & Borossilicato & $\mathrm{AcOH}$ anidro & 25 & 36 & $26 \%(\mathbf{8})$ \\
Alta Pressão & $\alpha$-santonina & Borossilicato & $\mathrm{H}_{2} \mathrm{O} / \mathrm{MeCN}(9: 7)$ & 25 & 36 & $37 \%(\mathbf{1 0})$ e $12 \%(\mathbf{9})$ \\
Alta Pressão & $\alpha$-santonina & Borossilicato & $\mathrm{AcOH} / \mathrm{H}_{2} \mathrm{O}(1: 1)$ & 25 & 36 & $32 \%(\mathbf{1 0})$ e $44 \%(\mathbf{9})$ \\
Alta Pressão & $\alpha$-santonina & Borossilicato & $\mathrm{MeCN}$ anidra & 25 & 36 & $39 \%(\mathbf{1 2})$ \\
Baixa Pressão & $\alpha$-santonina & Quartzo & $\mathrm{MeCN}$ anidra & 15 & 6 & $76 \%(\mathbf{1 1})$ \\
Baixa Pressão & $\alpha$-santonina & Quartzo & $\mathrm{MeCN}$ anidra & 70 & 2 & $76 \%(\mathbf{1 1})$ \\
Baixa Pressão & $\alpha$-santonina & Quartzo & $\mathrm{MeCN}$ anidra $_{2}$ & 70 & 24 & $100 \%(\mathbf{1 2})$ \\
Baixa Pressão & lumissantonina & Quartzo & $\mathrm{Et}_{2} \mathrm{O}$ anidro & 15 & 12 & $100 \%(\mathbf{1 2})$ \\
\hline
\end{tabular}


pressão, mantendo o sistema resfriado a $15^{\circ} \mathrm{C}$. Nessas condições foi isolada apenas a lactona 11 em 76\% de rendimento. A influência da temperatura sobre essa reação foi avaliada irradiando-se a $\alpha$-santonina nas mesmas condições, mas deixando o sistema aquecer até $70{ }^{\circ} \mathrm{C}$, o que resultou no mesmo rendimento de $\mathbf{1 1}$, mas com apenas $2 \mathrm{~h}$ de reação. Ao se repetir a reação por um tempo mais prolongado $(24 \mathrm{~h})$, a $\alpha$-santonina foi quantitativamente transformada na lactona 12 (Tabela 1). A lumissantonina (11) pode também ser obtida irradiando-se a $\alpha$-santonina em solução de dioxano ou em álcool etílico. ${ }^{22}$

Segundo Fisch e Richards, ${ }^{23}$ a formação do ácido 9 envolve a participação dos intermediários 11 e 12, conforme apresentado no Esquema 2.<smiles>CC1=C2[C@@H]3C(C)=C4[C@@H](C)C(=O)O[C@@H]4[C@@H]3CCC2(C)C=CC1=O</smiles>
$\alpha$-santonina

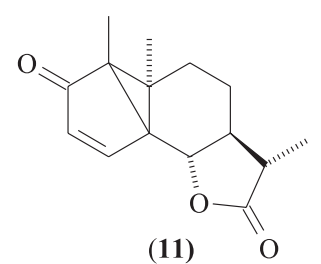

(12)

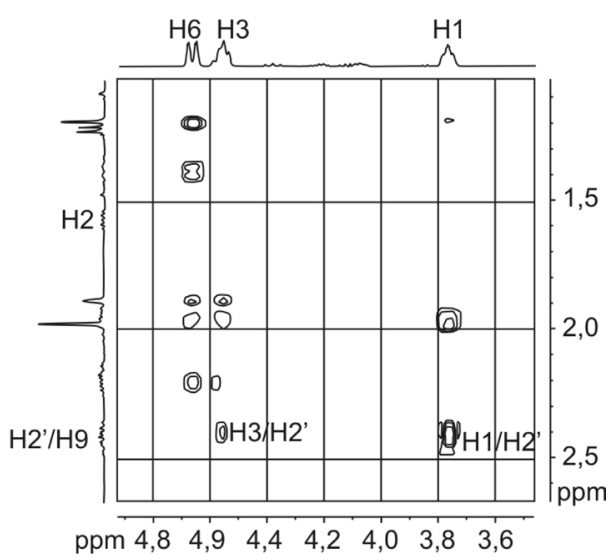

Figura 3. Mapa de contornos NOESY do composto 13

tadas por Zhang et al.. ${ }^{17}$ No espectro de RMN de ${ }^{13} \mathrm{C}$ do composto 13 observou-se uma mudança no deslocamento químico do sinal referente ao carbono $3(\delta=206,9$ para $\delta=77,9)$, evidenciando a redução do grupo carbonila. Dados de $\mathrm{RMN}$ de ${ }^{13} \mathrm{C}$ não foram descritos por Zhang et al.. ${ }^{17}$

Similarmente ao caso do composto 13, o álcool 14 apresentou a hidroxila no carbono $\mathrm{C}-3$ na face $\beta$, conforme depreendido pela análise do mapa de contornos NOESY, onde se observam as mesmas correlações em destaque na Figura 3.

\section{Ensaios biológicos}

Dos compostos submetidos à avaliação da atividade fitotóxica em placa de Petri na concentração de $4 \times 10^{-4} \mathrm{M}, \mathbf{9}, \mathbf{1 0}, 12$ e 14 estimularam o crescimento radicular das plântulas de pepino (Tabela 2). Os demais compostos causaram inibição, sendo que o composto 8 provocou a maior inibição $(48,1 \%)$. O álcool 13 causou menor efeito inibitório $(11,2 \%)$ que a cetona $\mathbf{8}$. Esse resultado, em parte, pode ser entendido considerando que grupos que podem reagir com nucleófilos estão associados à atividade fitotóxica. ${ }^{6}$ Dessa forma, o fato do composto 8 ter causado maior atividade inibitória, pode estar

Tabela 2. Fitotoxicidade da $\alpha$-santonina e dos compostos 8, 9, 10, 12,13 , e 14, na concentração de $4 \times 10^{-4} \mathrm{M}$, sobre o desenvolvimento do sistema radicular de Cucumis sativus e Sorghum bicolor, em placa de Petri, 72 h após semeado

\begin{tabular}{lcccc}
\hline & \multicolumn{2}{c}{$\begin{array}{c}\text { Comprimento da } \\
\text { radícula }(\mathrm{cm})\end{array}$} & \multicolumn{2}{c}{$\begin{array}{c}\text { Inibição da } \\
\text { radícula }(\%)^{* *}\end{array}$} \\
\hline Controle & $3,8 \mathrm{f}$ & $2,5 \mathrm{a}$ & 0,0 & 0,0 \\
$\alpha$-santonina & $2,7 \mathrm{gh}$ & $0,4 \mathrm{cde}$ & 27,9 & 84,0 \\
8 & $2,0 \mathrm{~h}$ & $0,4 \mathrm{cde}$ & 48,1 & 84,0 \\
9 & $6,0 \mathrm{a}$ & $0,4 \mathrm{cde}$ & $-56,8$ & 84,0 \\
10 & $4,8 \mathrm{bcde}$ & $1,0 \mathrm{~b}$ & $-25,4$ & 60,0 \\
12 & $5,6 \mathrm{ab}$ & $0,7 \mathrm{bc}$ & $-48,2$ & 72,0 \\
13 & $3,3 \mathrm{fg}$ & $0,4 \mathrm{cde}$ & 11,2 & 84,0 \\
14 & $5,3 \mathrm{abc}$ & $0,6 \mathrm{~cd}$ & $-39,8$ & 76,0 \\
\hline $\mathrm{CV}(\%)$ & 7,5 & 8,9 & - & - \\
\hline
\end{tabular}

*Médias seguidas por uma mesma letra na mesma coluna, não diferem entre si pelo teste de Tukey a 5\% de probabilidade. ** \% de inibição em relação ao tratamento controle. 
relacionado ao fato dele apresentar em sua estrutura um grupo cetona $\alpha, \beta$-insaturado que funciona como aceptor de Michael (ausente em 13), reagindo com grupos $\mathrm{SH}$ de enzimas. ${ }^{26}$ Foi observado também aumento no estímulo do crescimento para 14 (39,8\%) quando comparado com o composto $\mathbf{1 0}(25,4 \%)$. Os resultados obtidos nesse ensaio permitiram estabelecer a correlação estrutural preliminar dos derivados da $\alpha$-santonina com a atividade herbicida. Os guaianolídeos 8 e 13 provocaram inibição à raiz do pepino enquanto 10 e 14 estimularam o crescimento. Esses resultados podem estar associados à presença do grupo acetil no carbono C-10 nos guaianolídeos 8 e $\mathbf{1 3}$.

O composto 12 estimulou o crescimento das raízes do pepino em $48,2 \%$, enquanto a $\alpha$-santonina inibiu em $27,9 \%$. Embora ambas as moléculas possuam um esqueleto eudesmanolídeo e grupo capaz de atuar como receptor de Michael, tal diferença de atividade pode estar associada à baixa estabilidade de $\mathbf{1 2}$ quando armazenado na forma de óleo. ${ }^{21}$ Portanto, esse composto pode ter se degradado durante o período de armazenagem ou mesmo durante a realização dos ensaios biológicos. Nos bioensaios utilizando o sorgo como planta-teste verificou-se que todos os compostos, quando aplicados na concentração de $4 \times 10^{-4} \mathrm{M}$, apresentaram atividade fitotóxica, inibindo o crescimento radicular das plântulas de 60,0 a 84,0\% (Tabela 2).

Os compostos 8,9 e 13, e a $\alpha$-santonina foram os que apresentaram maior atividade inibitória e apesar dos diferentes esqueletos carbônicos os efeitos foram de $84,0 \%$.

A cetona $\mathbf{8}(84,0 \%)$ e o álcool $13(84,0 \%)$ apresentaram a mesma atividade inibitória ao passo que o diol 14 (76\%) apresentou maior atividade que a cetona $10(60 \%)$ em avaliações com o sorgo.

\section{CONCLUSÕES}

Com o objetivo de avaliar o potencial fitotóxico de lactonas sesquiterpênicas em Cucumis sativus e Sorghum bicolor foram preparados 7 derivados da $\alpha$-santonina. Cinco compostos foram preparados pela reação fotoquímica da $\alpha$-santonina variando o solvente, o reator (quartzo ou borossilicato) e a fonte de radiação (lâmpada de mercúrio de alta ou baixa pressão). Os álcoois 13 e 14 foram preparados, respectivamente, pela redução dos compostos $\mathbf{8}$ e 10 com boroidreto de sódio.

Quanto à atividade biológica dos compostos sintetizados, a lactona $\mathbf{8}$ apresentou a maior atividade fitotóxica tanto para pepino (48,1\% de inibição das raízes) quanto para sorgo (84,0\% de inibição das raízes). A diferença deste composto para os demais é a presença simultânea do grupo acetil no carbono 10 e do grupo carbonila $\alpha, \beta$ insaturado. Nenhum dos outros compostos avaliados apresentou a mesma combinação de grupos funcionais. Tais grupos podem reagir com os tióis das enzimas presentes nas plantas, resultando na atividade fitotóxica.

De modo geral, o sorgo foi mais susceptível aos compostos avaliados, enquanto o pepino teve seu crescimento radicular estimulado por algumas das moléculas preparadas $(\mathbf{9}, \mathbf{1 0}, 12$ e 14).

\section{AGRADECIMENTOS}

Ao CNPQ e à FAPEMIG pelo apoio financeiro e bolsas de mestrado (F. F. P. Arantes) e de pesquisa (L. C. A. Barbosa, A. J. Demuner); à CAPES pela bolsa de mestrado (W. A. Saliba).

\section{REFERÊNCIAS}

1. Duke, S. O.; Romagni, J. G.; Dayan, F. E.; Crop Protection 2000, 19, 583.

2. Duke, S. O.; Dayan, F. E.; Kagan, I. A.; Baerson, S. R. Em New Discoveries in Agrochemicals; Clark, J. M.; Ohkawa, H., eds.; ACS: New York, 2005.

3. Vyvyan, J. K.; Tetrahedron 2002, 58, 1631.

4. Macías, F. A.; Torres, A.; Molinillo, J. M. G.; Varela, R. M.; Galindo, J. C. G.; Pest. Manag. Sci. 2007, 63, 327.

5. Macías, F. A.; Fernández, A.; Varela, R. M.; Molinillo, J. M. G.; Torres, A.; Alves, P. L. C. A.; J. Nat. Prod. 2006, 69, 795.

6. Macías, F. A.; Torres, A.; Molinillo, J. M. G.; Varela, R. M.; Castellano, D.; Phytochemistry 1996, 43, 1205.

7. Barbosa, L. C. A.; Costa, A. V.; Veloso, D. P.; Lopes, J. L. C.; Terrones, M. G. H.; King-Diaz, B.; Lotina-Hensen, B.; Z. Naturforsch. C 2004, 59, 803; Costa, A. V.; Barbosa, L. C. A.; Lopes, J. L. C.; Veloso, D. P.; Magn. Reson. Chem. 2000, 38, 675.

8. Barbosa, L. C. A.; Demuner, A. J.; Borges, E. E. L.; Mann, J.; J. Braz. Chem. Soc. 1997, 8, 19; Demuner, A. J.; Barbosa, L. C. A.; Veloso, D. P.; Howarth, O. W.; Aust. J. Chem. 1998, 51, 61; Demuner, A. J.; Barbosa, L. C. A.; Veloso, D. P.; J. Agric. Food Chem. 1998, 46, 1173; Costa, A. V.; Barbosa, L. C. A.; Demuner, A. J.; Silva, A. A.; J. Agric. Food Chem. 1999, 47, 4807; Costa, A. V.; Barbosa, L. C. A.; Lopes, J. L. C.; Piló-Veloso, D.; Magn. Res. Chem. 2000, 38, 675; Barbosa, L. C. A.; Demuner, A. J.; Maltha, C. R. A.; Silva, P. S.; Silva, A. A; Quim. Nova 2003, 26, 655; Barbosa, L. C. A.; Maltha, C. R. A.; Demuner, A. J.; Filomeno, C. A.; Silva, A. A.; Quim. Nova 2004, 27, 241; Barbosa, L. C. A.; Alvarenga, E. S.; Demuner, A. J.; Virtuoso, L. S.; Silva, A. A.; Chem. Biodiversity 2006, 3, 553; Barbosa, L. C. A.; Rocha, M. E.; Teixeira, R. R.; Maltha, C. R. A.; Forlani, G.; J. Agric. Food Chem. 2007, 55, 8562; Teixeira, R. R.; Barbosa, L. C. A.; Forlani, G.; Piló-Veloso, D.; Carneiro, J. W. de M.; J. Agric. Food Chem. 2008, 56, 2321.

9. Picman, A. K.; Biochem. Syst. Ecol. 1986, 14, 255.

10. Perrin, D. D.; Armarego, W. L. F.; Purification of Laboratory Chemicals, $3^{\text {rd }}$ ed., Pergamon Press: New York, 1988.

11. Barbosa, L. C. A.; Alvarenga, E. S.; Demuner, A. J.; Figueiredo, R.; Silva, A. A.; Pest. Manag. Sci. 2003, 59, 1043.

12. Gomes, F. P.; Curso de Estatística Experimental, $3^{\text {a }}$ ed., Nobel: Piracicaba, 1990.

13. Barton, D. H. R.; Mayo, P.; Shafiq, M.; J. Chem. Soc. 1958, 140, 140.

14. Greene, A. E.; J. Am. Chem. Soc. 1980, 102, 5337.

15. Bargues, V.; Blay, G.; Cardona, L.; García, B.; Pedro, J. R.; Tetrahedron 1998, 54, 1845

16. Blay, G.; Bargues, V.; Cardona, L.; García, B.; Pedro, J. R.; Tetrahedron 2001, 57, 9719.

17. Zhang, W.; Luo, S.; Fang, F.; Chen, Q.; Hu, H.; Jia, X.; Zhai, H.; J. Am. Chem. Soc. 2005, 127, 18.

18. Asher, J. D. M.; Sim, G. A.; Proc. Chem. Soc. 1962, 111.

19. Barton, D. H. R.; Miki, T.; Pinhey, J. T.; Wells, R. J.; Proc. Chem. Soc. 1962, 112.

20. Barton, D. H. R.; De Mayo, P.; Shafiq, M.; J. Chem. Soc. 1957, 929

21. Zimmerman, H. E.; Schuster, D. I.; J. Am. Chem. Soc. 1962, 84, 4527.

22. Arigoni, D.; Bosshard, H.; Bruderer, H.; Büchi, G.; Jeger, O.; Krebaum, L. J.; Helv. Chim. Acta 1957, 180, 1732.

23. Fisch, M. H.; Richards, J. H.; J. Am. Chem. Soc. 1963, 85, 3029.

24. Fisch, M. H.; Richards, J. H.; J. Am. Chem. Soc. 1968, 90, 1553.

25. Chapman, O. L .; Englert, L. F.; J. Am. Chem. Soc. 1963, 85, 3028.

26. Macías, F. A.; Galindo, J. C. G.; Massanet, G. M.; Phytochemistry 1992, $31,1969$. 